\title{
Overview of the High-Level Trigger Electron and Photon Selection for the ATLAS Experiment at the LHC
}

A. Gesualdi Mello, A. dos Anjos, S. Armstrong, J. T. M. Baines, C. P. Bee, M. Biglietti, J. A. Bogaerts, M. Bosman, B. Caron, P. Casado, G. Cataldi, D. Cavalli, G. Comune, P. Conde Muiño, G. Crone, D. Damazio, A. De Santo, M. Diaz Gomez, A. Di Mattia, N. Ellis, D. Emeliyanov, B. Epp, S. Falciano, H. Garitaonandia, S. George, V. Ghete, R. Goncalo, J. Haller, S. Kabana, A. Khomich, G. Kilvington, J. Kirk, N. Konstantinidis, A. Kootz, A. J. Lankford, A. Lowe, L. Luminari, T. Maeno, J. Masik, C. Meessen, R. Moore, P. Morettini, A. Negri, N. Nikitin, A. Nisati, C. Osuna, C. Padilla, N. Panikashvili, F. Parodi, E. Pasqualucci, V. Perez Reale, J. L. Pinfold, P. Pinto, Z. Qian, S. Resconi, S. Rosati, C. Sanchez, C. Santamarina, D. A. Scannicchio, C. Schiavi, E. Segura, J. M. de Seixas, S. Sivoklokov, A. Sobreira, R. Soluk, E. Stefanidis, S. Sushkov, M. Sutton, S. Tapprogge, S. Tarem, E. Thomas, F. Touchard, G. Usaib, B. Venda Pinto, A. Ventura, V. Vercesi, T. Wengler, P. Werner, S. J. Wheeler, F. J. Wickens, W. Wiedenmann, M. Wielers, and G. Zobernig

\begin{abstract}
The ATLAS experiment at the Large Hadron Collider (LHC) will face the challenge of efficiently selecting interesting candidate events in $p p$ collisions at $14 \mathrm{TeV}$ center-of-mass energy, whilst rejecting the enormous number of background events. The High-Level Trigger (HLT $=$ second level trigger and Event Filter), which is a software based trigger will need to reduce the level-1 output rate of $\approx 75 \mathrm{kHz}$ to $\approx 200 \mathrm{~Hz}$ written out to mass storage. In this talk an overview of the current physics and system performance of the HLT selection for electrons and photons is given. The performance has been evaluated using Monte Carlo simulations and has been partly demonstrated in the ATLAS testbeam in 2004. The efficiency for the signal channels, the rate expected for the selection, the global data preparation and execution times will be highlighted. Furthermore, some physics examples will be discussed to demonstrate that the triggers are well adapted for the physics programme envisaged at the LHC.
\end{abstract}

Index Terms-ATLAS experiment, combined test beam results, online selection of electrons and photons.

\section{INTRODUCTION}

$\mathbf{T}$ HE ATLAS (A Toroidal LHC Apparatus) detector [1] is one of the two mayor multi-purpose detectors currently under construction at the Large Hadron Collider (LHC). Its inner elements are tracking detectors enclosed in a solenoidal magnet of around $2 \mathrm{~T}$ in the central part. From the inside to the outside, it consists of pixel detectors, silicon strip detectors (SCT) and transition radiation detectors (TRT).

The tracking detectors are surrounded by an electromagnetic calorimeter based on liquid Argon technology and a hadronic calorimeter based on LAr in the end-caps and a sampling calorimeter with an active part of scintillators (Tile) in the barrel. The global detector dimensions (diameter $22 \mathrm{~m}$, length $42 \mathrm{~m}$ ) are defined by a large air-core muon spectrometer.

The physics program envisaged ranges from the search for the Higgs boson, which is the last missing particle within the

Manuscript received June 17, 2005; revised May 30, 2006.

Please see the Acknowledgment section of this paper for the author affiliations.

Digital Object Identifier 10.1109/TNS.2006.882049

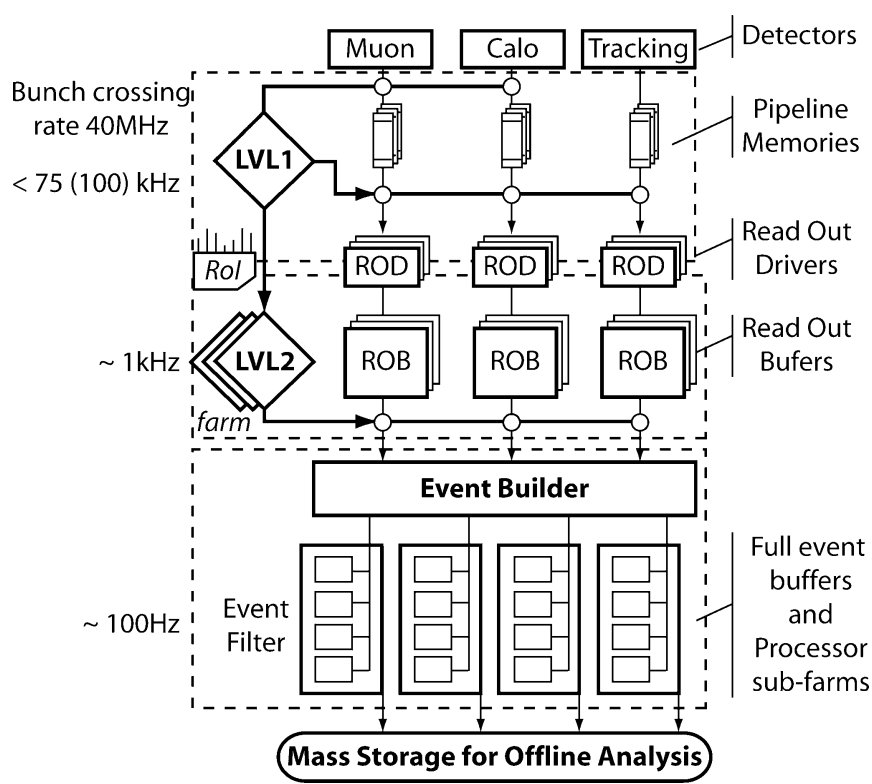

Fig. 1. Block diagram of the ATLAS Trigger/DAQ system.

Standard Model (SM), searches for physics beyond the SM such as supersymmetric particles, new additional $\mathrm{W}$ and $\mathrm{Z}$ bosons, etc., precision SM studies, like measurements of the $t$ quark and $W$ boson masses, and detecting possible unexpected signals from unpredicted physics scenarios.

At LHC, protons will collide at a center of mass energy of $14 \mathrm{TeV}$, with a design luminosity of $10^{34} \mathrm{~cm}^{-2} \mathrm{~s}^{-1}$. The corresponding $40 \mathrm{MHz}$ bunch crossing rate (with an average of $\approx 23$ superimposed events) and the huge amount of read-out channel $\left(\approx 10^{8}\right)$ outline the challenge of the ATLAS Trigger and Data Acquisition (TDAQ) system.

\section{THE ATLAS TDAQ SYSTEM OVERVIEW}

The ATLAS Trigger and Data Acquisition (TDAQ) system must be able to select and store events at a bunch crossing rate of $40 \mathrm{MHz}$. The required data reduction factor, equivalent to 


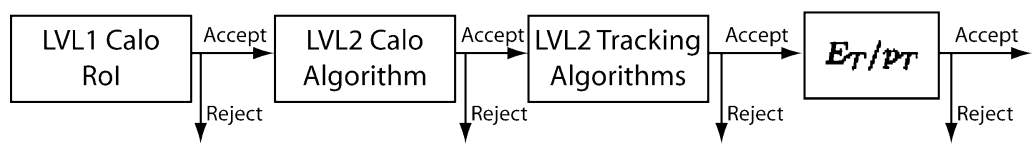

Fig. 2. Trigger event selection diagram. Step-based execution of sequences of seeded algorithms.

a rejection factor of about six orders of magnitude, is achieved on-line via a data acquisition system organized in three different trigger levels (LVL1, LVL2 and Event Filter), as shown in Fig. 1.

Each level refines the decisions made at the previous one and, where necessary, applies additional selection criteria. The time available for event processing increases in each level. This allow the use of an increasing amount of information to either accept or reject the event. The hardware-based First Level Trigger (LVL1) performs a preliminary rejection using only reduced granularity data coming from the calorimeters and the muon detectors. It operates within a $2 \mu$ s latency, producing a maximum output rate of $75 \mathrm{kHz}$, upgradable to $100 \mathrm{kHz}$.

The High-Level Triggers (LVL2 and Event Filter) [7], implemented on two different commodity component farms, provide a further reduction factor of about $10^{3}$. Reconstruction at LVL2, seeded by information collected at LVL1, can exploit the information at full granularity from all ATLAS subdetectors. LVL2 reconstruction is performed by a parallel data processing of one or more geometrical regions identified at LVL1. These regions, called Regions of Interest (RoI), correspond to about 2\% of the total event size. Event selection is designed to provide on average an output rate of $1 \mathrm{kHz}$. The LVL2 decision must be taken with a mean processing time of $10 \mathrm{~ms}$. This execution time is one of the major contraints of the LVL2 reconstruction algorithms.

\section{The Electron and Photon Selection Goal}

Events with electrons and photons in the final state are important signatures for many physics analysis envisaged at the LHC, as electrons and photons are relatively easy to identify and trigger on. For example, $H \rightarrow 4 e$ of $H \rightarrow \gamma \gamma$ lead to a final state containing isolated electrons and/or photons, which provide very clean signatures.

Electron and photon reconstruction mainly exploits data coming from the Electromagnetic calorimeter and the Inner Detector (ID) tracking systems. As described in detail in the next section [8], electrons can be identified in the calorimeter by looking at the transverse shower shapes and the leakage into the hadronic calorimeters. For electrons, a track is searched by matching of the calorimeter cluster. In the case of photons, conversions photons can be searched for. As will be shown, this will results in the required rejection of $10^{3}$ in the HLT while retaining around $80 \%$ of electrons and photons.

\section{A. Implementation at the HLT}

The LVL2 $e / \gamma$ trigger is the starting point for the formation of LVL2 electron and photon trigger objects. The $e / \gamma$ trigger procedure at the HLT is guided by the Region of Interest mechanism. In particular, LVL2 reconstruction uses information on the transverse energy and the direction of the electromagnetic clusters selected by the LVL1 trigger. The LVL2 trigger refines the LVL1 information using full-granularity information from the calorimeters. The LVL2 trigger also profits from improved, though not final, calibrations and thresholds.

First, the energy and position measurements obtained at LVL1 are refined. The measurement of $E_{T}$ at LVL2 results in sharper thresholds and allows tighter $E_{T}$ cuts. Then, the leakage into the hadronic calorimeter is evaluated and variables related to the transverse shower shape in the electromagnetic calorimeter are used to perform preliminary particle identification. If a candidate is found to be consistent with an electron, track reconstruction is performed in the ID. In the next step, cluster-to-track association is done using $(\eta, \phi)$ matching criteria, achieving further rejection against fake candidates. In case the matching was successful, the $E_{T} / p_{T}$ ratio between the transverse energy measured in the EM calorimeter and the transverse momentum of the corresponding ID track is evaluated for particle identification as well as the match in $\Delta \eta$ and $\Delta \phi$ between the calorimeter cluster and the extrapolated track. Fig. 2 shows a simplified block diagram of $e / \gamma$ trigger steps described. In the case of photon candidates, reconstructed EM clusters undergo tighter shower shape cuts.

After each step in the selection a hypothesis algorithm is called and the event processing is only continued if the $e / \gamma$ candidate is still compatible with an electron or photon. If the objects under analysis fulfill the required signatures the event and its LVL2 result are passed to the Event Filter (EF). In the EF information on the complete event is available, along with more precise calibrations and alignment constants. As at LVL2 electrons and photon selection can be seeded by the improved direction as found at LVL2. Even if selection at the EF follows the same scheme described for LVL2 operation, the looser timing $(\approx 1 \mathrm{sec})$ constraints enable to employ more sophisticated reconstruction algorithms such as bremsstrahlung recovery for electrons and conversion reconstruction for photons.

\section{LVL2 ALGORITHMS}

The challenging ATLAS on-line environment imposes strong requirements on the design of the LVL2 system [9]. In the following, the present view of the algorithms needed to implement LVL2 selection is given. Several options for using the detector information in the best possible way are taken into account, and hence more than one algorithm can accomplish a defined task. This will allow the implementation of a robust, flexible and redundant selection scheme, which will be studied with present and future simulations.

\section{A. T2Calo}

T2Calo is a clustering algorithm for electromagnetic(EM) showers. It is seeded by Level-1 EM trigger RoI positions. This algorithm takes calibrated calorimeter cells as input and provides discriminating variables to separate isolated EM objects 
from jets using shower-shape quantities and the leakage into the hadronic calorimeter. The improved measurement of the electron/photon candidate allows results in a sharper thresholds and helps to select efficiently candidates above a certain energy threshold defined by the trigger menu item.

The first step of T2Calo is the refinement of the Level-1 position. The highest energetic cell is searched for in the second layer of the EM calorimeter (typically more than $70 \%$ of the cluster energy is deposited in this sampling). The electron/photon cluster is then built around this seed cell in a window of $\Delta \eta \times \Delta \phi=0.075 \times 0.125$. Subsequently the cluster energy will be corrected to account for the leakage outside the window.

The position $\left(\eta_{1}, \phi_{1}\right)$ of the highest energy cell is calculated using the energy-weighted cluster position in this second sampling.

\section{B. IDSCAN}

IDSCAN [10], [11] is a track-reconstruction package for LVL2. It takes as input Space Points found in the Pixel and SCT Detectors. A series of sub-algorithms (Z-Finder, Hit Filter, Group Cleaner, Track Fitter) then process these inputs and output Tracks and the Space Points associated with them.

\section{SiTrack}

SiTrack [12] is another track-reconstruction package for LVL2 which takes Pixel and SCT Space Points as input. The output are fitted reconstructed Tracks. Each track stores the pointers to the Space Points used to build it. SiTrack is implemented as a single main algorithm and executes a user-defined list of subalgorithms.

\section{TRT-LUT}

TRT-LUT is a LVL2 algorithm for track reconstruction in the TRT [13], [14]. The algorithm takes as input Hits in the TRT. The algorithmic processing consists of Initial Track Finding, Local-Maximum Finding, Track Splitting, Track Fitting and Final Selection. It outputs the Hits used and Tracks with their parameters, which are $\phi, p_{T}$, electric charge $Q$ and the track curvature $C$.

\section{E. TRTxKalman}

The TRTxKalman [15] utilizes only the information from the TRT part of the Inner Detector. The core of the algorithm is a set of utilities from the offline reconstruction package xKalman [6] for reconstructing tracks in the TRT detector. It is based on the Hough-transform (histogramming) method. At the initialization step of the algorithm, a set of trajectories in the $\phi-R(Z)$ space is calculated for the barrel and endcap parts of the TRT. The real value of the magnetic field is taken into account at each hit in the straw along the track when calculating the track trajectories.

\section{LVL2 $e / \gamma$ TRIGGER EFFICIENCY}

We have several algorithms addressing the building of Space Points and the tracking in the ID, with several individual benefits and charactristics.

The performance of the $e / \gamma$ trigger menus has been evaluated on Monte Carlo simulated samples for which the detector
TABLE I

RATES AND EFFICIENCIES FOR THE SINGLE ELECTRON TRIGGER Selecting Electrons With a $p_{T}>25 \mathrm{GEV}$

\begin{tabular}{|l|l|l|}
\hline & Eff (\%) & Rate \\
\hline LVL2 Calo & 97.3 & $1.9 \mathrm{kHz}$ \\
\hline LVL2 ID & 93.1 & $395 \mathrm{~Hz}$ \\
\hline LVL2 IDCalo & 91.0 & $170 \mathrm{~Hz}$ \\
\hline EF Calo & 90.0 & $125 \mathrm{~Hz}$ \\
\hline EF ID & 84.9 & $75 \mathrm{~Hz}$ \\
\hline EF IDCalo & 79.8 & $40 \mathrm{~Hz}$ \\
\hline
\end{tabular}

TABLE II

Timing PERFORMANCE OF THE $e / \gamma$ SLICE

\begin{tabular}{|l|l|}
\hline & Time $(\mathrm{ms} / 8 \mathrm{GHz}$ CPU RoI $)$ \\
\hline T2Calo & 4.0 \\
\hline IDSCAN & 7.4 \\
\hline Total & 6.0 \\
\hline
\end{tabular}

response has been simulated in detail by GEANT. Results are given in terms of the efficiency for the real electron and photon signals and of the expected output rates, directly related to the rejection power for fake candidates.

As an example Table I shows the efficiency and rejection rate for the trigger menu selecting single isolated electrons with a transverse energy $\left(E_{T}\right)$ exceeding $25 \mathrm{GeV}$ (e25i) at initial luminosity $\left(L=2 \times 10^{33} \mathrm{~cm}^{-2} \mathrm{~s}^{-1}\right)$. Errors, as also in the following, only take into account the statistical uncertainty contribution. It should be noted that the uncertainties in the QCD dijet cross-sections at the LHC are about a factor 2. Results have been evaluated on a simulated single electron with $p_{T}=25 \mathrm{GeV}$ with a flat distribution over the full tracking rapidity range $|\eta|<2.5$. The efficiencies and rates are evaluated, after each HLT selection step, with respect to a LVL1 output efficiency of $\approx 95 \%$ and a LVL1 EM cluster rate of $12 \mathrm{kHz}$.

The preliminary HLT $e / \gamma$ results on timing are shown in Table II. Data access and preparation, referred to as unpacking, corresponds to a critical timing consuming step for LVL2 algorithms. The timing results for QCD jet events events at $L=$ $2 \times 10^{33} \mathrm{~cm}^{-2} \mathrm{~s}^{-1}$, were measured per RoI and scaled to a $8 \mathrm{GHz}$ machine.

The total timing performance presented in Table II were calculated taking into account the rejection power after each selection step (see Table I). Around $90 \%$ of the time is currently spent in the unpacking of the data. In order to improve unpacking timing measurements, a new data access approach is presently under development.

\section{EXPERIENCE With Combined Test BEAm DATA}

In 2004, elements of all ATLAS sub-detectors were aligned along a beam line to test the overall sub-detector performance as well as the combined performance for electrons, photons jets and muons [17], as depicted in Fig. 3.

During the test beam period part of the trigger system was as well tested. To validate the LVL2 algorithms and the electron selection strategy the recorded CTB data is being analysed and $e / \pi$ separation has been studied.

Preliminary results of LVL2 Calorimeter (T2Calo) electron selection algorithm for $50 \mathrm{GeV}$ electrons and pions, are shown in Fig. 4. Electron efficiency and pion fake rate calculation using 


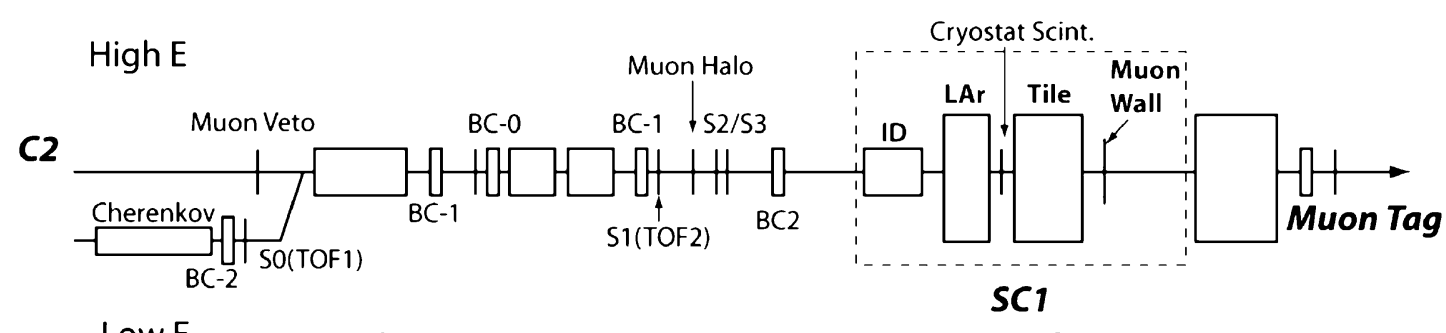

Low $\mathrm{E} \quad$ Using beam detectors to get a clean sample of $\mathrm{e} / \pi$

Fig. 3. Block diagram of the $e / \pi$ separation study with 2004 CTB data using the LVL2 calorimeter and tracking algorithms.

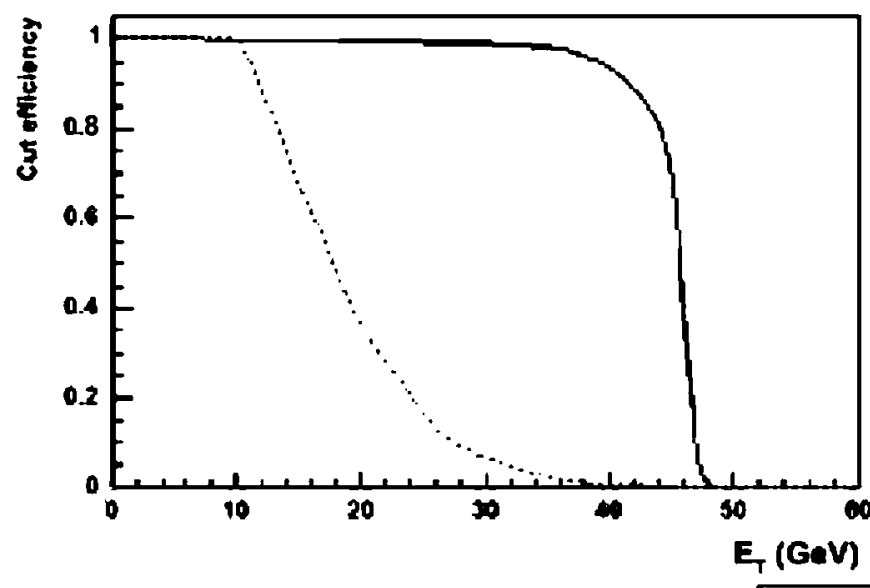

(a)

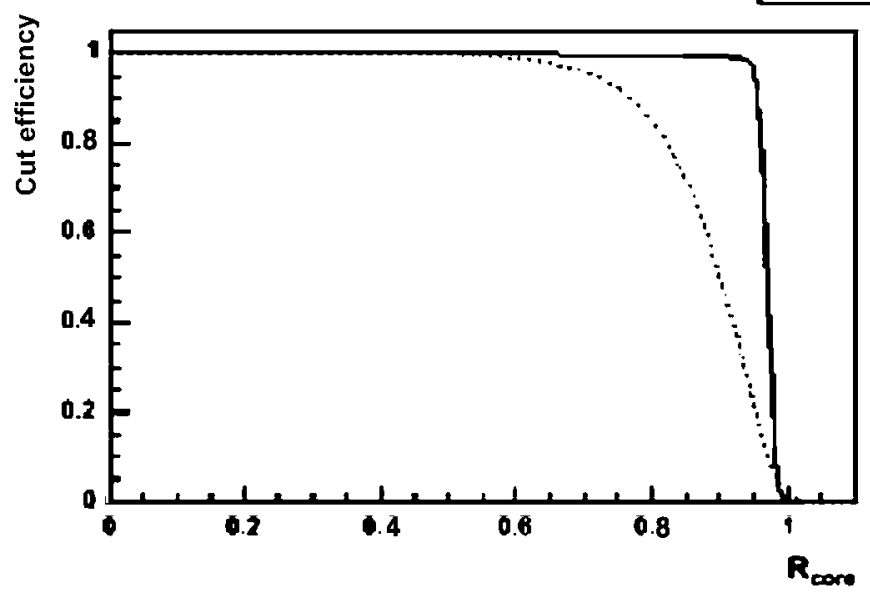

(c)

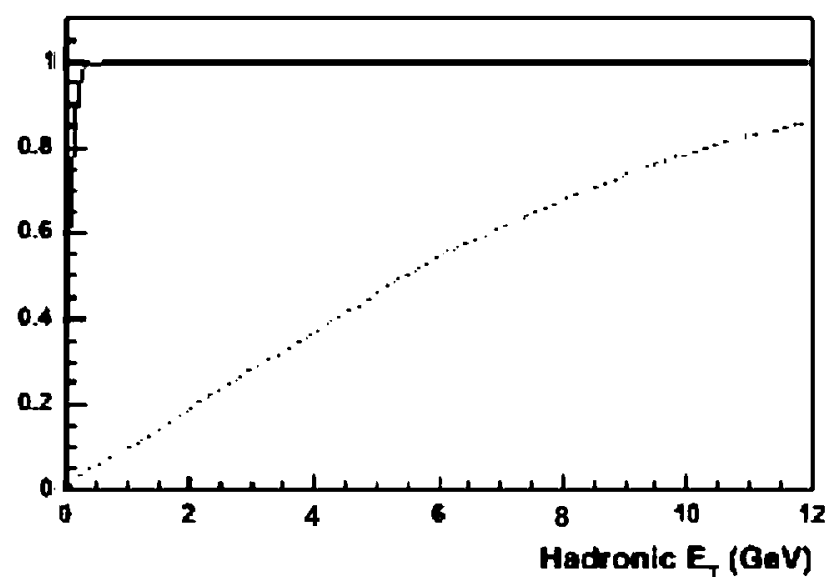

(b)

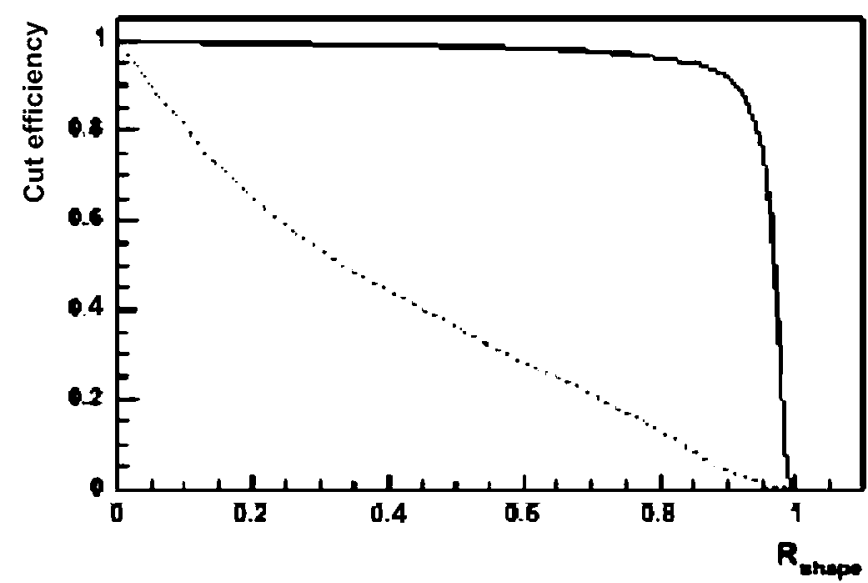

(d)

Fig. 4. Study $e / \pi$ separation with 2004 CTB data using the LVL2 calorimeter and tracking algorithms. (a) Cut efficiency at the first step of T2Calo algorithm. (b) Cut efficiency at the second step of T2Calo. (c) Cut efficiency at the third step of T2Calo. (d) Cut efficiency at the last step of T2Calo.

L2 trigger calorimeter are presented in Table III. The electron and pion beams contain (depending on energy) a certain fraction of pion, muon and electrons. Using the beam instrumentation and the information of the TRT the electron and pion beam was cleaned up.

\section{CONCLUSION}

In this paper we presented an overview of the HLT $e / \gamma$ selection for the ATLAS Experiment at the LHC. The electron selection efficiency for single-e with pile-up for LVL2, at $25 \mathrm{GeV}$
TABLE III

EFFICIENCY AND FAKE RATE RESULTS OF LVL2 CALORIMETER TRIGGer at $50 \mathrm{GEV}$

\begin{tabular}{|l|l|l|}
\hline & The electron efficiency & Pion fake rate \\
\hline LVL2 calorimeter cuts & $98.82 \pm 0.18$ & $1.13 \pm 0.14$ \\
\hline \multicolumn{3}{|c}{ Electron purity $=97 \%$} \\
\hline \multicolumn{2}{|c|}{ Pion purity $=100 \%$} \\
\hline
\end{tabular}

with a luminosity of $2 \times 10^{33} \mathrm{~cm}^{-2} \mathrm{~s}^{-1}$, was $\approx 97 \%$ with a rate of $1.9 \mathrm{kHz}$ for LVL2 Calo and $\approx 91 \%$ for LVL2 ID-Calo with 
$170 \mathrm{~Hz}$ of rate. Estimates of time and rates meet the requirements at start-up, i.e., $\approx 5 \%$ of LVL2 CPU power. The electron identification efficiency using real data from the CTB (Combined Test Beam) 2004 at $50 \mathrm{GeV}$ was $98.8 \pm 0.2 \%$ with a pion fake rate of $1.13 \pm 0.14 \%$. Efficiency and fake rate results are consistent with previous LAr studies using 2002 test beam data. This has been an important step to validate the selection architecture chosen in a real on-line environment.

\section{ACKNOWLEDGMENT}

A. G. Mello and J. M. de Seixas are with the Universidade Federal do Rio de Janeiro, COPPE/EE, Rio de Janeiro, Brazil.

A. dos Anjos, J. Haller, J. Kirk, W. Wiedenmann, and G. Zobernig are with the Department of Physics, University of Wisconsin, Madison, Wisconsin, USA.

S. Armstrong, D. Damazio, and T. Maeno are with the Brookhaven National Laboratory (BNL), Upton, New York, USA.

J. T. M. Baines, D. Emeliyanov, F. J. Wickens, and M. Wielers are with the Rutherford Appleton Laboratory, Chilton, Didcot, U.K.

C. P. Bee, C. Meessen, Z. Qian, and F. Touchard are with the Centre de Physique des Particules de Marseille, IN2P3-CNRS-Université d'Aix-Marseille 2, France.

M. Biglietti is with the Dipartimento di Fisica dell'Università degli studi di Napoli 'Federico II' e I.N.F.N., Napoli, Italy.

J. A. Bogaerts, P. Conde Muiño, N. Ellis, C. Padilla, P. Pinto, S. Rosati, C. Santamarina, A. Sobreira, T. Wengler, and P. Werner are with the CERN, Geneva, Switzerland.

M. Bosman, P. Casado, H. Garitaonandia, C. Osuna, C. Sánchez, E. Segura, and S. Sushkov are with the Institut de Física d'Altes Energies (IFAE), Univerdidad Autónoma de Barcelona, Barcelona, Spain.

B. Caron, R. Moore, J. L. Pinfold, and R. Soluk are with the University of Alberta, Edmonton, Canada.

G. Cataldi and A. Ventura are with the Dipartimento di Fisica dell'Università di Lecce e I.N.F.N., Lecce, Italy.

D. Cavalli and S. Resconi are with the Dipartimento di Fisica dell'Università di Milano e I.N.F.N., Milan, Italy.

G. Comune is with the Department of Physics and Astronomy, Michigan State University, East Lansing, MI 48824, USA.

G. Crone, N. Konstantinidis, E. Stefanidis, and M. Sutton are with the Department of Physics and Astronomy, University College London, London, U.K.

A. De Santo, S. George, R. Goncalo, G. Kilvington, and A. Lowe are with the Department of Physics, Royal Holloway, University of London, Egham, U.K.

M. D. Gomez is with the Section de Physique, Université de Genève, Switzerland.

A. Di Mattia, S. Falciano, L. Luminari, A. Nisati, and E. Pasqualucci are with the Dipartimento di Fisica dell'Università di Roma 'La Sapienza' e I.N.F.N., Rome, Italy.

B. Epp and V. Ghete are with the Institut für Experimentalphysik der Leopold-Franzens Universität, Innsbruck, Austria.

S. Kabana, V. Perez Reale, and E. Thomas are with the Laboratory for High Energy Physics, University of Bern, Switzerland.

A. Khomich is with the Lehrstuhl für Informatik V, Universität Mannheim, Mannheim, Germany.

A. Kootz is with the Fachbereich Physik, Bergische Universität Wuppertal, Germany.

A. J. Lankford is with the University of California at Irvine, Irvine, CA USA.

J. Masik is with the Institute of Physics, Academy of Sciences of the Czech Republic, Prague, Czech Republic.
P. Morettini, F. Parodi, and C. Schiavi are with the Dipartimento di Fisica dell'Università di Genova e I.N.F.N., Genoa, Italy.

A. Negri, D. A. Scannicchio, and V. Vercesi are with the Dipartimento di Fisica Nucleare e Teorica dell'Università di Pavia e INFN, Pavia, Italy.

N. Nikitin and S. Sivoklokov are with the Institute of Nuclear Physics, Moscow State University, Moscow, Russia.

N. Panikashvili and S. Tarem are with the Department of Physics, Technion, Haifa, Israel.

S. Tapprogge is with the Institut für Physik, Universität Mainz, Mainz, Germany.

G. Usaib is with the Dipartimento di Fisica dell'Università di Pisa e I.N.F.N. Pisa, Italy.

B. Venda Pinto is with the CFNUL-Universidade de Lisboa, Faculdade de Ciências, Lisbon, Portugal.

S. J. Wheeler is with the University of Alberta, Edmonton, Canada, and also with the University of California at Irvine, Irvine, CA USA.

\section{REFERENCES}

[1] ATLAS: Technical Proposal for a General-Purpose pp Experiment at the LHC, CERN/LHCC/94-43, 1994, ATLAS Collaboration.

[2] ATLAS Detector and Physics Performance Technical Design Report, CERN/LHCC/99-14, ATLAS-TDR-014, 1999, ATLAS Collaboration.

[3] Inner Detector Technical Design Report, CERN/LHCC/97-16, ATLAS-TDR-4, 1997, ATLAS Inner Detector Community.

[4] Calorimeter Performance Technical Design Report, CERN/ LHCC/96-40, 1996, ATLAS Collaboration.

[5] ATLAS Muon Spectrometer Technical Design Report, CERN/ LHCC/97-22, 1997, ATLAS Muon Collaboration.

[6] ATLAS High-Level Trigger, Data Acquisition and Controls Technical Design Report, CERN/LHCC/2003-022, ATLAS-TDR-016, 2003, ATLAS HLT/DAQ/DCS Group.

[7] ATLAS High-Level Triggers, DAQ and DCS Technical Proposal, CERN/LHCC/2000-017, 2000, ATLAS Collaboration.

[8] Implementation and Performance of the High-Level Trigger Electron and Photon Selection for the ATLAS Experiment at the LHC, ATLASCOM-DAQ-2005-012, 2005, C. Schiavi on behalf of the ATLAS HighLevel Trigger Group.

[9] S. González, T. Hansl-Kozanecka, and M. Wielers, Selection of High- $p_{T}$ Electromagnetic Clusters by the Second Level Trigger of ATLAS ATLAS Internal Note, ATL-DAQ-2000-002, 2000.

[10] H. Drevermann and N. Konstantinidis, Determination of the z Position of Primary Interactions in ATLAS ATLAS Internal Note, ATL-DAQ2002-014, 2002.

[11] H. Drevermann and N. Konstantinidis, Algorithms to Select Space Points of Tracks From Single Primary Interactions in ATLAS ATLAS Internal Note, ATL-COM-DAQ-2003-040, 2003

[12] M. Cervetto, P. Morettini, F. Parodi, and C. Schiavi, iTrack: A Level-2 Track Reconstruction Algorithm Based on Silicon Detectors ATLAS Internal Note ATL-COM-DAQ-2003-025, 2003.

[13] J. T. M. Baines, Global Pattern Recognition in the TRT for B-Physics in the ATLAS Trigger ATLAS Internal Note, ATL-DAQ-99-012, 1999.

[14] M. Sessler and M. Smizanska, Global Pattern Recognition in the TRT for the ATLAS LVL2 Trigger ATLAS Internal Note, ATL-DAQ-98120, 1998, 13-36.

[15] I. Gavrilenko, Description of Global Pattern Recognition Program (Kalman) ATLAS Internal Note ATLAS-INDET-97-165, 1997.

[16] S. González, B. G. Pineiro, and T. Shears, First Implementation of Calorimeter FEX Algorithms in the LVL2 Reference Software ATLAS Internal Note, ATL-DAQ-2000-020, 2000.

[17] S. Gadomski, "Deployment and use of the ATLAS DAQ in the combined test beam," IEEE Trans. Nucl. Sci., vol. 53, no. 4, pp. 2156-2161, Aug. 2006 